BNL-73463-2005-CP

\title{
Novel Method of Emittance Preservation in ERL Merging System in Presence of Strong Space Charge Forces
}

\author{
D. Kayran and V.N. Litvinenko \\ Presented at the Particle Accelerator Conference (PAC '05) \\ Knoxville, Tennessee \\ March 19-23, 2005
}

Collider-Accelerator Department

Brookhaven National Laboratory

P.O. Box 5000

Upton, NY 11973-5000

www.bnl.gov

Managed by

Brookhaven Science Associates, LLC

for the United States Department of Energy under

Contract No. DE-AC02-98CH10886 


\section{DISCLAIMER}

This work was prepared as an account of work sponsored by an agency of the United States : Government. Neither the United States Government nor any agency thereof, nor any of their employees, nor any of their contractors, subcontractors or their employees, makes any warranty, express or implied, or assumes any legal liability or responsibility for the accuracy, completeness, or any third party's use or the results of such use of any information, apparatus, product, or process disclosed, or represents that its use would not infringe privately owned rights Reference herein to any specific commercial product, process, or service by trade name, trademark, manufacturer, or "otherwise, does not necessarily: constitute or imply its endorsement, recommendation, or favoring by the United States Government or any agency thereof or its contractors or subcontractors. The views and opinions of authors expressed herein do not necessarily state or reflect those of the United States Government or any agency thereof. 


\title{
NOVEL METHOD OF EMITTANCE PRESERVATION IN ERL MERGING SYSTEM IN PRESENCE OF STRONG SPACE CHARGE FORCES*
}

\author{
D. Kayran, V.N: Litvinenko, BNL, Upton, NY 11973, U.S.A..
}

\section{Abstract:}

Energy recovery linacs (ERLs) are potential candidates for the high power and high brightness electron beams sources. The main advantages of ERL are that electron beam is generated at relatively low, energy, injected and accelerated to the operational energy in a linac, and after the use is decelerated in the same linac down to injection energy, and, finally, dumped: A merging.system, i.e. a system merging together high energy and low energy beams, is an intrinsic part of any ERL loop.: One of the challenges for generating high, charge, high brightness electron beams in an ERL is development of a merging system, : which provides achromatic condition for space charge dominated beam and which is compatible with the emittance compensation scheme. In this paper we present principles of operation of such merging systems : We also describe an example of such system; which : we call a Zigzag or a Z-system. We use a specific implementation of: the Z-system for: R\&D 'ERL. at: Brookhaven as the illustration......

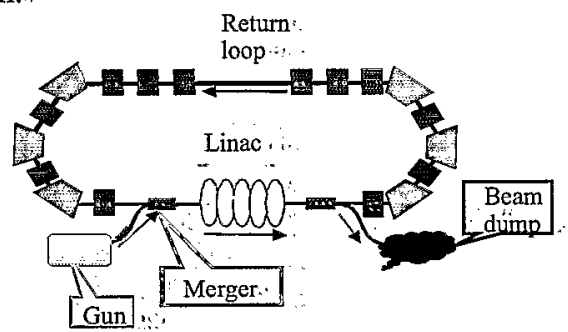

Figure 1.Schematics view of ERL.

\section{MOTIVATION}

Each ERL loop (Fig. 1) has at least one merging system; which includes dipoles. Any dipole is a source of coupling between $\left(\delta=\left(E-E_{o}\right) / E_{o}, \xi=s-v t\right)$ longitudinal: and $\left(\mathrm{x}, \mathrm{p}_{\mathrm{x}}\right)$ transverse phase space planes.

The space charge forces repel particles from each other. Hence, the particles in the head of the bunch gain energy, while the particles at the tail of the bunch lose energy (see Fig. 2). This effect is very significant for. low energy beams with high charge per bunch. Low injection energy (well below $10 \mathrm{MeV}$ ) is strongly desirable in high current: ERLs to lessen the radiation hazards and to reduce; requirements for $R F$ power.

The use of achromatic system for a merger will decouple the motion; : but only in the absence of the longitudinal space charge forces. In the presence of the space charge forces, the coupling resulting from the variation of the particle's energy can cause significant

*Work performed under the auspices of the U.S. Department of Energy and partially funded by the US Department of Defence.

"dkayran@bnl:gov growth of the transverse emittance in a traditional achromatic system:

In addition, the emittance compensation schemes [1] do : not allow using a strong focusing in a merger. This requirement limits even further the number of available merger schemes used for high charge, high brightness electron beams..

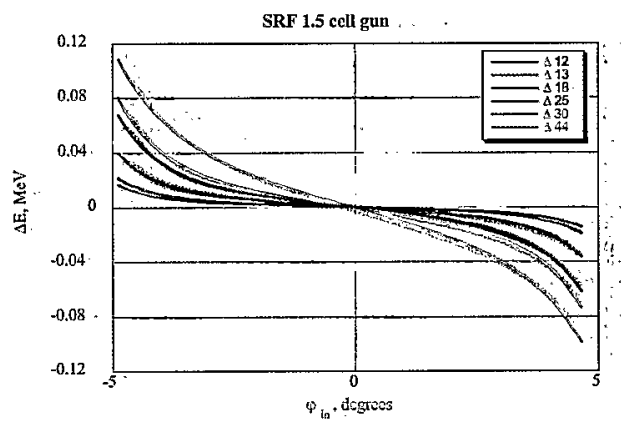

Figure 2:Variation of electron energy, $\Delta E=E(\mathrm{~s})-E(0)$, in a merger (caused by: the space charge forces of $1 . \mathrm{nC}$ bunch): is a function of its position in the bunch (shown in the units of a relative $703.75 \mathrm{MHz}$ RF phase). Different colours indicate different locations along the beam line, which indices: $(12,13,18,25,30,44)$ showing the element numbers in lattice file for PARMELA [2]. Fitting is defined by eq: (1):

One of the keys to a successful solution of the above problem is finding a correlation between electron's energy change and its location in the bunch. For example, Fig. 2 shows results of PARMELA [2] simulation of a 1 nC "bunch with initial "beer-can" distribution [3]. The other beam parameters at the cathode were: duration $10^{\circ}:$ (39.5 psec), radius $4 \mathrm{~mm}, 1.5$ cell $\mathrm{RF}$ : gun with energy gain of $3.7 \mathrm{MeV}$ : The energy change of the particle is defined by longitudinal electric field $\mathbf{E}$ :

$$
\frac{d E}{d s} \cong e \mathrm{E}(\zeta)
$$

where $\zeta_{-}$is longitudinal coordinate of the particle relative of the bunch center. The resulting dependencies (see Fig. 2) fit very well with analytical formula for the field of the homogeneously charged cylinder [3]:

$$
\mathrm{E}\left(\zeta_{0}\right)=\frac{2 Q}{r^{2} \cdot 2 l}\left(2 \zeta_{o}-\sqrt{r^{2}+\left(\zeta_{o}+l\right)^{2}}+\sqrt{r^{2}+\left(\zeta_{o}-l\right)}\right),(1)
$$

where $Q$ is the charge, $r$ is the radius and $l$ is the length of ... the beam.

The most importantly, the fit for the energy changes with the formula allows to separate variables and to express it as a function of initial longitudinal coordinates $\left(\delta_{o}, \zeta_{o}\right)$ and the azimuth $s$ along the orbit (Fig: 3): $\delta(s) \cong \delta_{o}+f\left(\zeta_{o}\right) \cdot\left(s+\alpha \cdot s^{2}\right)$. 
Thus, energy dependence vs $s$ for any electron is well described by its dependence on two parameters - the initial energy and the initial phase. Hence, in general case, we are seeking a 2-parameter dependence:

$$
\delta_{i}(s)=a_{i} \cdot g_{1}(s)+b_{i} \cdot g_{2}(s)
$$

where: $i$ is the index of the particle, $E_{i}$ is the energy of $\mathrm{i}^{\text {th }}$ particle, $a_{i}$ and $b_{i}$ are individual parameters for $\mathrm{i}^{\text {th }}$ particle (presumably some functions of the initial $\left(\delta_{i o}, \zeta_{i o}\right)$ ), $g_{l}(s)$ and $g_{2}(s)$ are the function of azimuth $s$, which are the same for all particles in the beam. It is important that results of following method are completely independent from the specific dependence of parameters $a_{i}$ and $b_{i}$ on $\left(\delta_{i o}, \zeta_{i o}\right) /$

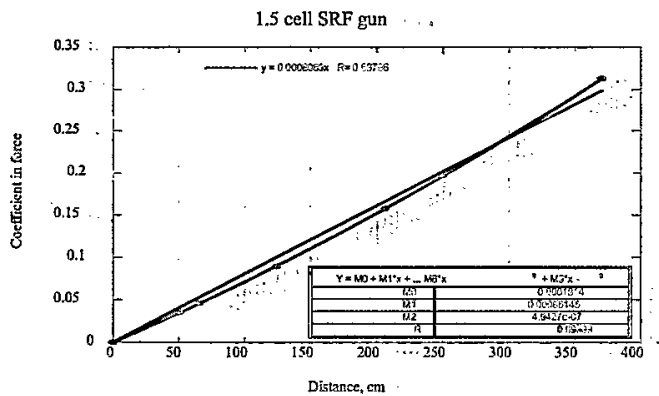

Figure 3. Dependence of the energy gain on the azimuth $s$. Red dots are the results of simulations; the blue lines. are linear and second order polynomial fits:

\section{CONCEPT:}

Horizontal betatron oscillations around the ideal trajectory are described : by well-known : homogeneous linear equation:

$$
\frac{d X}{d s} \equiv X^{\prime}=D(s): X(s) ; \quad D=\left[\begin{array}{cc}
0 & 1 \\
-K_{1}(s) & 0
\end{array}\right]
$$

where $X^{T}=\left[x, p_{x}\right]$ and $K_{l}(s)$ is defined by focusing strength of magnets and the space charge of the beam. The solution of equation (3) can be expressed in matrix form:

$$
X(s)=M(s) \cdot X(0)
$$

where $X(0)$ is initial transverse phase space coordinates: The $M(s)$ is the $2 \times 2$ transport matrix from 0 to $s$. which is satisfied conditions:

$$
M^{\prime}=D(s) \cdot M ; \operatorname{det} M=1 .
$$

For a particle with energy deviation $\delta(s)$ the equation of motion becomes inhomogeneous:

$$
\Psi^{\prime}(s)=D(s) \cdot \Psi(s)+K_{o}(s) \cdot \delta(s) \cdot\left[\begin{array}{l}
0 \\
1
\end{array}\right],
$$

where $K_{o}(s)=e B_{y}:$ Using $: \Psi(s)=M(s) \cdot A(s)$ :

$$
A^{\prime}=K_{o} \cdot \delta \cdot M^{-1} \cdot\left[\begin{array}{l}
0 \\
1
\end{array}\right] \Rightarrow A^{\prime}=K_{o} \cdot \delta \cdot\left[\begin{array}{c}
-m_{12} \\
m_{11}
\end{array}\right]
$$

and zero initial conditions $\Psi(o)=0$ one gets:

$$
A^{T}(s)=\left[-\int_{0}^{s} K_{o}\left(s^{\prime}\right) \cdot \delta\left(s^{\prime}\right) m_{12}\left(s^{\prime}\right) d s^{\prime}, \int_{0}^{s} K_{o}\left(s^{\prime}\right) \cdot \delta\left(s^{\prime}\right) m_{11}\left(s^{\prime}\right) d s^{\prime} \cdot\right]
$$

Hence, ... for the decoupling : of transverse and longitudinal motions (it is actually the condition on $4 \times 4$ symplectic matrix [5]) at the end of the system, $s_{f}$, one should request that for each electron two conditions are satisfied:

$$
A\left(s_{f}\right)=0 .
$$

Using parameterization (2); one can rewrite (5) in the form of four conditions:

$$
\begin{aligned}
& \int_{0}^{s} K_{o}\left(s^{\prime}\right) \cdot g_{1}(s) \cdot m_{11}\left(s^{\prime}\right) d s^{\prime}=0 ; \int_{0}^{s} K_{o}\left(s^{\prime}\right) \cdot g_{2}(s) \cdot m_{11}\left(s^{\prime}\right) d s^{\prime}=0 \\
& \int_{0}^{s} K_{o}\left(s^{\prime}\right) \cdot g_{1}(s) \cdot m_{12}\left(s^{\prime}\right) d s^{\prime}=0 ; \int_{0}^{s} K_{i}\left(s^{\prime}\right) \cdot g_{2}(s) \cdot m_{12}\left(s^{\prime}\right) d s^{\prime}=0 ;
\end{aligned}
$$

which: provide sufficient for the decoupling of transverse and longitudinal motion for all electrons within the bunch.

\section{System with bilateral symmetry (Zigzag)}

The simplest case of parameterization in eq: (2) is that of a "frozen".longitudinal motion:

$$
\delta^{\prime}=g\left(\zeta_{o}\right) \Rightarrow \delta_{i}(s)=\delta_{i o}+s \cdot g\left(\zeta_{i o}\right)
$$

which is a good approximation for variety: of the processes relevant to the space charge effects (see Fig. 3, where linear approximation fits $\mathrm{g}_{2}(\mathrm{~s})$ rather well, or see Refs. $[6,7]$ : where similar considerations were applied for: coherent synchrotron radiation effects);

The decoupling conditions in this case are:

$$
\begin{array}{ll}
\int_{0}^{s} K_{o}\left(s^{\prime}\right) \cdot m_{11}\left(s^{\prime}\right) d s^{\prime}=0 ; & \int_{0}^{s} K_{o}\left(s^{\prime}\right) \cdot s \cdot m_{11}\left(s^{\prime}\right) d s^{\prime}=0 ; \\
\int_{0}^{s} K_{o}\left(s^{\prime}\right) \cdot m_{12}\left(s^{\prime}\right) d s^{\prime}=0 ; & \int_{0}^{s} K_{o}\left(s^{\prime}\right) \cdot s \cdot m_{12}\left(s^{\prime}\right) d s^{\prime}=0 ;
\end{array}
$$

Let's consider a system, which we call Zigzag, with symmetrical focusing $K_{1}(s)=+K_{1}(s)$ and asymmetrical curvature $K_{o}(s)=-K_{o}(s)$ (see Fig. 4).

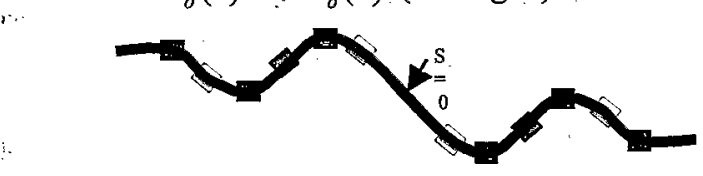

Figure 4. Schematic : of a Zigzag: green boxes are the dipoles, red and blue boxes are focusing and defocusing lenses...

The elements of the transport matrix for such systems are coupled by the conditions for the bilateral symmetry:

$$
\begin{aligned}
& M(-s)=\left[\begin{array}{cc}
1 & 0 \\
0 & -1
\end{array}\right] M(s)\left[\begin{array}{cc}
1 & 0 \\
0 & -1
\end{array}\right] . \\
& \Rightarrow m_{11}(-s)=m_{11}(s) ; m_{12}(-s)=m_{12}(s)
\end{aligned}
$$

Hence for Zigzag system two achromatic conditions from: (7) are automatically satisfied:

$$
\begin{aligned}
& K_{o}(-s) \cdot m_{11}(-s)=-K_{o}(s) \cdot m_{11}(s) \Rightarrow \int_{-L}^{T_{o}} K_{o}\left(s^{\prime}\right) \cdot m_{11}\left(s^{\prime}\right) d s^{\prime} \equiv 0 \\
& K_{o}(-s) \cdot(-s) \cdot m_{12}(-s)=-K_{o}(s) \cdot(s) \cdot m_{12}(s) \Rightarrow \int_{-L} K_{o}\left(s^{\prime}\right) \cdot m_{12}\left(s^{\prime}\right) s^{\prime} \cdot d s^{\prime} \equiv 0
\end{aligned}
$$

The rest of achromatic conditions (7) can be rewritten as:

$$
\int_{0}^{L} K_{j}\left(s^{\prime}\right) \cdot m_{12}\left(s^{\prime}\right) d s^{\prime}=0 ; \cdots \quad \int_{0}^{L} K_{o}\left(s^{\prime}\right) \cdot s: m_{11}\left(s^{\prime}\right) d s^{\prime}=0 .(8)
$$

Example: A simplest system consists of $2 \mathrm{~K}$ short dipoles (with bending angle $\theta_{k}:$ and position $: s_{k}$ each) without focusing in horizontal direction. In this case the elements 
of transport matrix are: $m_{11}=1, m_{12}=s$ and only one condition remains:

$$
\sum_{k=1}^{K} s_{k} \cdot \theta_{k}=0
$$

For $\mathrm{K}=2$ the condition (9) gives a simplest Zigzag with $s_{2}=2 s_{1}, \theta_{1}=-2 \theta_{2}[8]$ :

\section{RESULTS OF THE TESTS}

Detailed results of the test of the concept can be found in $[5,8]$ : Here we present only main results by comparing a traditional achromat (chicane) with a Z-system (see Fig. 4). To make a fair comparison, both systems have the same focusing strength and are made of chevron with 86 $\mathrm{cm}$ radii or curvature: Both configurations are achromatic for particle with constant energy. This resulted in following parameters:

ZigZag lattice: $10^{\circ}$ bend, $40 \mathrm{~cm}$ drift, $-20^{\circ}$ bend, 81.6 $\mathrm{cm}, 20^{\circ}$. bend, $40 \mathrm{~cm}$ drift, $: 10^{\circ}$ bend

Chicane lattice: $12.4^{\circ}$ bend, $47.5 \mathrm{~cm}$ drift, $-11.36^{\circ}$ bend; $96.6 \mathrm{~cm}, 11.36^{\circ}$ bend, $47.5 \mathrm{~cm}$ drift, $-12.4^{\circ}$ bend

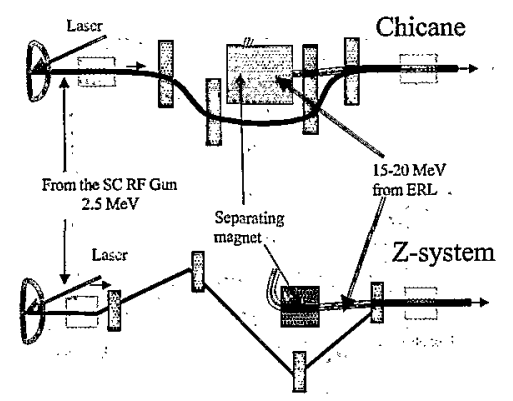

Figure 4. Schematics of traditional chicane and a Zigzagsystem for an ERL:

In the numerical test performed with PARMELA, a 1 . $\mathrm{nC}$ electron bunch from the $\cdot 1.5$-cell $\cdot \mathrm{RF}^{*}$ gun was propagated through the above merging: systems followed by a $15 \cdot \mathrm{MeV}-703.75 \mathrm{MHz}$. linac: The electron beam energy. at the gun exit : was $\gamma \mathrm{mc}^{2}=4.2 \mathrm{MeV}$. Initial beam:: has “"beer-can distribution":: duration of $12^{\circ}(47 \mathrm{psec})$ and: radius $4 \mathrm{~mm}$.

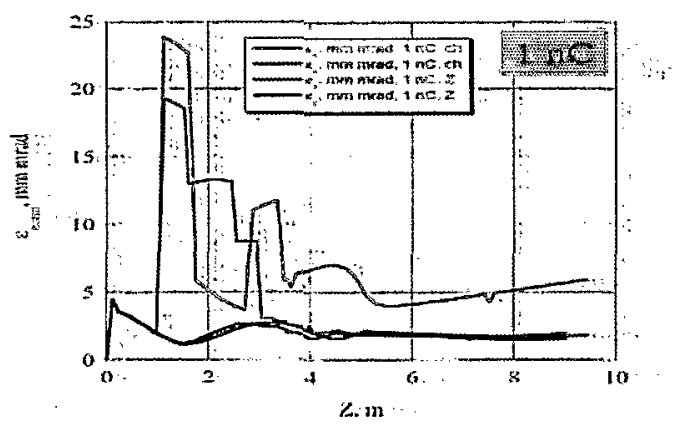

Figure 5: Results of PARMELA simulation for $1 \mathrm{nC}$.
Results of the PARMELA simulation are shown in Fig. 5. In both cases, for the chicane and the Z-system, vertical normalized emittances are equal to about $1.8 \mathrm{~mm} * \mathrm{mrad}$ at the linac exit, the indication of the equivalence of the systems for the process of emittance compensation. In contrast, the horizontal : emittance behavior is very different for two systems. After passing the Z-system horizontal $*$ emittance and vertical emittance have practically the same value, while the chicane results in: a doubling of the horizontal emittance.

\section{CONCLUSIONS}

We developed the new concept to the ERL merging system compatible with the emittance compensation schemes for generating high brightness electron beam. To our surprise this simple concept; some version of which were intuitively used previously. [6,7], works very well for many processes, including space "charge dominated. magnetized . beams ... [10] and" coherent . synchrotron ... radiation [6]:

\section{ACKNOWLEDGEMENTS}

The authors are grateful to Ilan Ben-Zvi for fruitful: discussions and for bring this problem to our attention; to Rama Calaga, Xiangyun - Chang and Jörg Kewisch: for their help with the simulation of the RF gun and with additional tools for PARMELA simulation, and for the test of the concept with magnetized beams.

\section{REFERENCES}

[1] J. B. Rosenzweig and L. Serafini, Phys. Rev. E 49, 1599 (1994).....

[2] L.. M: Young, J. H: Billen, PARMELA documentation, LAUR-96-1835.

[3]: "Beer-can". distribution is an uniformly charged cylinder.

[4] V.V. Batygin; I.N. Taptygin, Collection of E\&M problems, Second Edition, Nauka, Moscow; 1970

[5] D. "Kayran, V. Litvinenko, Novel merging system preserving the emittance of high brightness high charge beams in ERLs, in preparation.

D. Kayran, :V.N. Litvinenko, Optimum merger for an ERL; ERL 2005, March 2005, Newport News, VA.

[6] David H. Dowell, Compensation of bend-plane emittance growth in a 180 Degree Bend, PAC97

[7]. Ryoichi Hajima -First-Order Matrix : Approach to the Analysis of Electron Beam Emittance GrowthCaused by Coherent Synchrotron Radiation, Jpn.. J. Appl. Phys. Vol.' 42 (2003) pp. L 974-L 976

[8] V. N. Litvinenko et al,, Proceedings of the 2004 FEL Conference, p. 570 http://accelconf.web.cern.ch/AccelConf/f04;:

[9] I. Ben-Zvi at al., Extremely High Current, High-Brightness Energy Recovery Linac, these proceedings.

[10]-Electron : Cooling of RHIC, I. Ben-Zvi : et al,; these proceedings

Electron Beam Generation and Transport for the RHIC Electron Cooler, J. Kewisch et al:, these proceedings. 